\title{
ROS1-ADGRG6: a case report of a novel ROS1 oncogenic fusion variant in lung adenocarcinoma and the response to crizotinib
}

\author{
Shuguang $\mathrm{Xu}^{1}$, Wenxian Wang ${ }^{2}$, Chunwei $\mathrm{Xu}^{3}$, Xingliang $\mathrm{Li}^{4}$, Junhui Ye $\mathrm{F}^{5}$, Youcai Zhu ${ }^{4}$ and Ting Ge $\mathrm{G}^{*}$
}

\begin{abstract}
Background: ROS1 rearrangements are validated drivers in lung cancer, which have been identified in a small subset (1-2\%) of patients with non-small cell lung cancer (NSCLC). To date, 18 fusion genes of ROS1 have been identified in NSCLC. The ALK inhibitor (crizotinib) exhibits therapeutic effect against ROS1-rearranged NSCLC. Nextgeneration sequencing (NGS) technology represents a novel tool for ROS1 detection that covers many fusion genes.

Case presentation: A 55-year-old female with EGFR mutation (L858R) was diagnosed with lung adenocarcinoma, who was responsive to first-generation EGFR-tyrosine kinase inhibitor (TKI). Afterwards, she developed acquired resistance accompanied with a ROS1 rearrangement. A NGS assay showed that the tumor had a novel ROS1ADGRG6 rearrangement generated by the fusion of exons of 1-33 of ROS1 on chr6: q22.1 to exons of 2-26 of ADGRG6 on chr6: q24.2. The patient was obviously responsive to crizotinib.

Conclusion: We firstly identified ROS1-ADGRG6 fusion variant in NSCLC by NGS, which should be considered in further ROS1 detecting assays.
\end{abstract}

Keywords: Lung adenocarcinoma, NGS, ROS1 rearrangement

\section{Background}

Morbidity and mortality of lung cancers has been gradually increased during the past several decades [1]. The ROS proto-oncogene 1, receptor tyrosine kinase (ROS1) gene is proved to be a valuable therapeutic target in patients with non-small cell lung cancer (NSCLC). It has been established that solid tumors have unstable genomes, and many fusions are caused by genetic instability. The prevalence of ROS1 rearrangements is estimated in 1-2\% of NSCLC patients [2]. Up to date, a total of 18 ROS1 fusion genes have been reported in lung cancer, including CD74, SLC34A2 and GOPC [3-5]. All ROS1 gene fusions harbor the ROS1 kinase domain, with CD74-ROS1 being the most common fusion partner. Studies have shown that these alterations frequently lead to activation of signaling

\footnotetext{
*Correspondence: gt1103@163.com

${ }^{6}$ Department of Respiratory, Ningbo Medical Center Lihuili Hospital, Ningbo, Zhejiang 315010, People's Republic of China

Full list of author information is available at the end of the article
}

pathways that are critical for carcinogenesis and progression, such as MAPK and PI3K/AKT pathways. Moreover, these fusions play a prognostic role in lung cancer [6]. For example, ROS1 fusion-positive patients with lung cancer have poorer disease-free survival (DFS) than those fusionnegative patients [7].

Crizotinib is an anaplastic lymphoma kinase (ALK)/ ROS1/MET inhibitor. Based on efficacy and safety data from a clinical trial, crizotinib has become the first targeted agent approved by the FDA for the treatment of advanced ROS1-rearranged NSCLC $[8,9]$. In addition to FISH, IHC, and PCR, next-generation sequencing (NGS) has emerged as a new diagnostic approach for detection of ROS1 rearrangements in recent years.

In this case, we identified a novel ROS1 fusion gene in a lung adenocarcinoma patient. We also report that the patient was sensitive to treatment with ROS1-directed tyrosine kinase inhibitors (TKIs).

(c) The Author(s). 2019 Open Access This article is distributed under the terms of the Creative Commons Attribution 4.0 International License (http://creativecommons.org/licenses/by/4.0/), which permits unrestricted use, distribution, and reproduction in any medium, provided you give appropriate credit to the original author(s) and the source, provide a link to the Creative Commons license, and indicate if changes were made. The Creative Commons Public Domain Dedication waiver (http://creativecommons.org/publicdomain/zero/1.0/) applies to the data made available in this article, unless otherwise stated. 


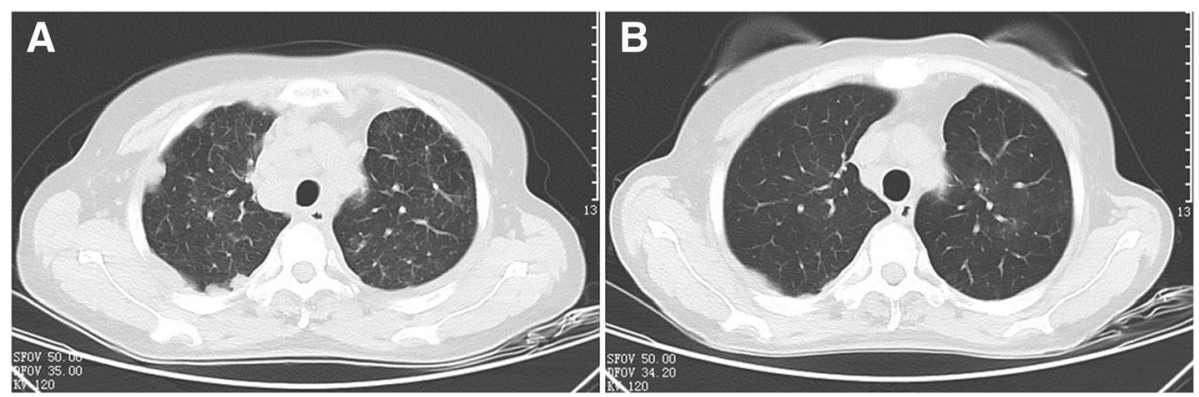

Fig. 1 Computed tomography (CT) scans before (a) and after (b) crizotinib therapy

\section{Case presentation}

A 55-year-old female was referred to our hospital in April 2016 with a 2-month history of cough and phlegm. A computed tomography (CT) scan revealed multiple nodules in the left lower lung (Fig. 1a). She underwent thoracoscopic surgery for radical resection of lung tumors. Hematoxylin and eosin (H\&E) staining revealed a typical morphology for adenocarcinoma cells (Fig. 2). The patient relapsed in November 2016 and was initially treated with gefitinib due to detection of an EGFR mutation (L858R) without ROS1 fusion by the captured targeted next-generation sequencing 381 panel. Although a decrease in tumor size was obtained in a short-time period, long-term effects were not achieved. Subsequently, she underwent chemotherapy (pemetrexed and carboplatin for 6 cycles, pemetrexed alone for 2 cycles) in December 2016. Then, the patient was treated with oral afatinib administration in August 2017, and combined treatment with docetaxel and carboplatin for 5 cycles in November 2017. However, the response was inadequate. After three months, chest $\mathrm{CT}$ scan images indicated an increase in tumor size. A NGS analysis of the hydrothorax revealed a novel ROS1ADGRG6 rearrangement, as shown in Fig. 3a (3D Medicines, Shanghai China). This novel ROS1-ADGRG6 rearrangement was generated the fusion of exons of 1-33 of
ROS1 on chr6: q22.1 to exons of 2-26 of ADGRG6 on chr6: q24.2. The predicted ROS1-ADGRG6 protein product contained 3075 amino acids comprising the $\mathrm{N}$-terminal amino acids 1-1853 of ROS1 and C-terminal amino acid 1-1222 of ADGRG6 (Fig. 3b). Thus, the patient received oral crizotinib therapyin April 2018. After 1 month, a chest CT scan showed a decrease in tumor size and the patient achieved a partial response to crizotinib (Fig. 1b). During crizotinib therapy, there were no adverse events, such as rashes, cordis damage, and gastrointestinal reactions. Thus far, the disease remains stable and she is still under treatment with crizotinib after 6 months.

\section{Discussion and conclusion}

Currently, 18 fusion partners of ROS1 fusions have been reported in lung cancer. A functional investigation has shown the oncogenic potential of ROS1 fusions. For example, ROS1 fusions results in transformation of NIH3T3 in vitro and tumorigenicity in vivo $[10,11]$. Transgenic mice harboring EZR-ROS1 in the lung alveolar epithelial cells develop bilateral lung adenocarcinomas [12, 13]. Indeed, ROS1-ADGRG6 rearrangement has not been previously reported in lung cancer, thus this is the first report of a novel ROS1 fusion variant. Given that the patient was initially responded to

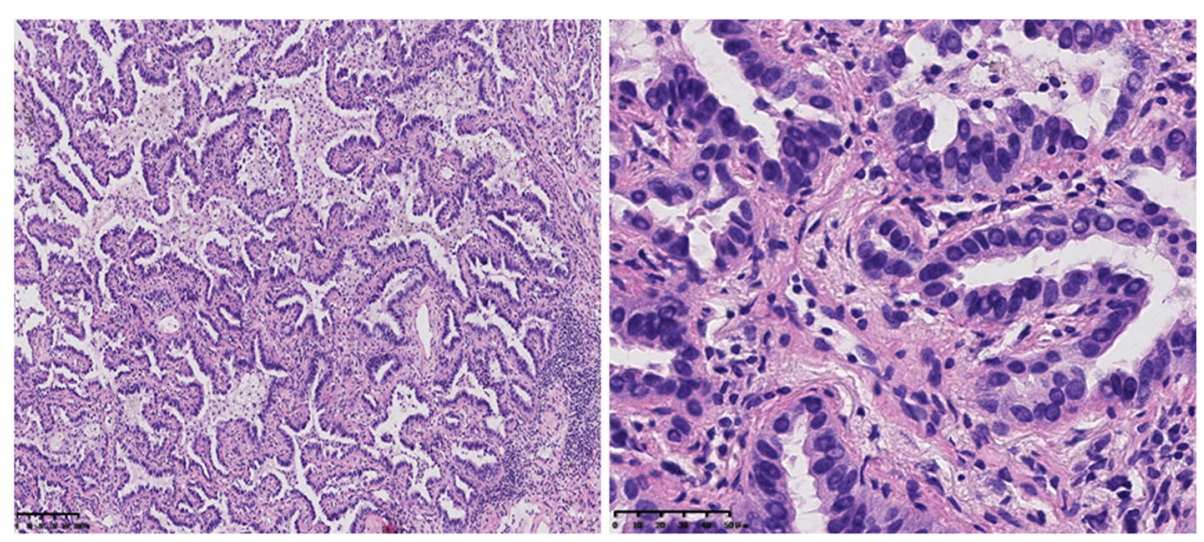

Fig. 2 Surgery of brain tumor showed adenocarcinoma lung cancer ( $\mathrm{HE} \times 10$, left; $\mathrm{HE} \times 40$, right) 


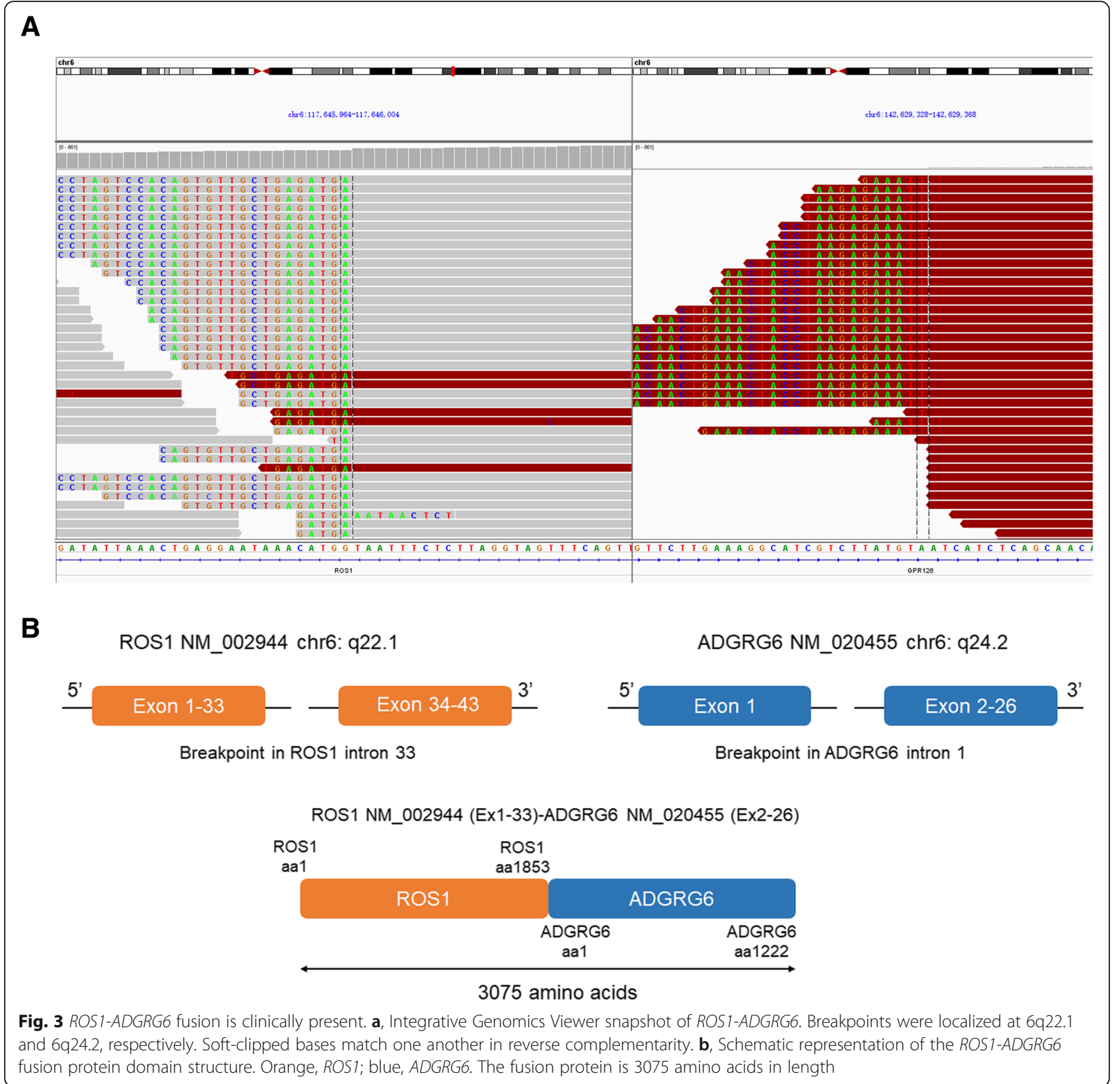

gefitinib, but later developed acquired resistance, we proposed this novel ROS1 fusion may be responsible for the acquired EGFR-TKI resistance.

Adhesion G protein-coupled receptor G6 (ADGRG6 [also referred to as GPR126]) is located on chromosome 6q24.2 and contains 28 exons, while ROS1 is located on chromosomes 6q22.1. ADGRG6 is a member of the adhesion G protein-coupled receptor family, which consists of a seventransmembrane domain and a long $\mathrm{N}$-terminal region involved in cell adhesion [14, 15]. Thus, it remains to be determined whether or not patients with ROS1-rearranged lung cancer and the ROS1-ADGRG6 fusion exhibit unique clinicopathologic manifestations, such as metastasis.
Although crizotinib was approved to treat advanced lung cancer with ROS1 rearrangement, there are currently no approved companion diagnostic assays to detect ROS1 rearrangements in NSCLC. Traditional methods (including FISH and IHC) have limitations, such as they both depend on diagnostic expertise. Another diagnostic method, i.e., RT-PCR, is unable to detect novel chromosomal rearrangements $[15,16]$. By contrast, NGS allows for detection of both known and previously unreported ROS1 rearrangements, as in this case.

Malignant pleural effusions (MPEs) are often present in advanced lung cancer patients. Given that MPEs contain tumor cells and biomarkers, they are considered to 
be an alternative to tumor tissues for detection of genetic mutation and fusions. FISH and RT-PCR have been successfully applied to detect EGFR mutations and $A L K$ rearrangements in MPEs [7, 17]. In our case, the ROS1 fusion was detected in a MPE using NGS, suggesting that evaluation of a MPE represents an alternative and feasible method to detect gene fusions in NSCLC.

There are some limitations in our present study. Firstly, this is only a case report and more cases are needed to analyze the correlation of ROS1-ADGRG6 and clinical parameters, such as overall survival and progression-free survival. Secondly, the biological function of ROS1-ADGRG6 should be further investigated using cell lines and animal models after molecular manipulation of ROS1-ADGRG6.

In summary, the present case indicated that ROS1$A D G R G 6$ fusion may underlie the acquisition of resistance against EGFR-TKI and suggested an important role for the diagnostic application of NGS in precision medicine.

\section{Abbreviations}

ADGRG6: adhesion G protein-coupled receptor G6; ALK: Anaplastic lymphoma kinase; NGS: Next-generation sequencing; TKIs: Tyrosine kinase inhibitors

\section{Acknowledgements}

Not applicable.

\section{Authors' contributions}

SX wrote the work; SX, WW, CX, XLL, JY, YZ, TG contributed to writing and revising the work for important intellectual content and have given final approval of the version. All authors made substantial contributions to the conception of the work and have given agreement to be accountable for all aspects of the work. All authors read and approved the final manuscript.

\section{Funding}

The Medical Scientific Research Foundation of Zhejiang Province of China (2019RC027) and the Science and Technology Scheme of Ningbo (2016A610198) supported this work. The funding had no role in the design of the study and collection, analysis, and interpretation of data and in writing the manuscript.

\section{Availability of data and materials}

For patients' privacy, the patient information is publicly inaccessible.

\section{Ethics approval and consent to participate}

The authors declare they have observed appropriate ethical guidelines and legislation in writing the case report. Consent to participate was obtained from the patient.

\section{Consent for publication}

The authors confirm that written informed consent for publication of case details and any accompanying images were provided by the patient. A copy of the signed, written informed consent for publication form is available for review by the editor

\section{Competing interests}

The authors declare they have no competing interests.

\section{Author details}

'Department of Respiratory Medicine, Ningbo Medical Center Lihuili Eastern Hospital, Ningbo, China 315010, People's Republic of China. ${ }^{2}$ Department of Chemotherapy, Zhejiang Cancer Hospital, Hangzhou, Zhejiang 310022,

People's Republic of China. ${ }^{3}$ Department of Pathology, Fujian Cancer
Hospital, Fujian Medical University Cancer Hospital, Fuzhou, Fujian 350014, People's Republic of China. ${ }^{4}$ Department of Thoracic Disease Diagnosis and Treatment Center, Zhejiang Rongjun Hospital, Jiaxing, Zhejiang 314000, People's Republic of China. ${ }^{5}$ Department of Respiratory, Sanmen People's Hospital of Zhejiang, Zhejiang 317100, People's Republic of China. ${ }^{6}$ Department of Respiratory, Ningbo Medical Center Lihuili Hospital, Ningbo, Zhejiang 315010, People's Republic of China.

Received: 30 November 2018 Accepted: 17 July 2019

Published online: 05 August 2019

\section{References}

1. Siegel RL, Miller KD, Jemal A. Cancer statistics, 2018. CA Cancer J Clin. 2018; 68:7-30.

2. Chong CR, Bahcall M, Capelletti M, et al. Identification of existing drugs that effectively target NTRK1 and ROS1 rearrangements in lung cancer. Clin Cancer Res. 2017;23:204-13.

3. Network CGAR. Comprehensive molecular profiling of lung adenocarcinoma. Nature. 2014;511:543-50.

4. Gounder MM, Hakimi AA, Harding JJ, et al. Mutational landscape of metastatic cancer revealed from prospective clinical sequencing of 10,000 patients. Nat Med. 2017:23:703-13.

5. Zhu YC, Zhou YF, Wang WX, et al. CEP72-ROS1: a novel ROS1 oncogenic fusion variant in lung adenocarcinoma identified by next-generation sequencing. Thorac Cancer. 2018;9:652-5.

6. Schram AM, Chang MT, Jonsson P, et al. Fusions in solid tumours: diagnostic strategies, targeted therapy, and acquired resistance. Nat Rev Clin Oncol. 2017;14:735-48.

7. Kim MH, Shim HS, Kang DR, et al. Clinical and prognostic implications of ALK and ROS1 rearrangements in never-smokers with surgically resected lung adenocarcinoma. Lung Cancer. 2014;83:389-95.

8. Shaw AT, Ou SH, Bang YJ, et al. Crizotinib in ROS1-rearranged non-small-cell lung cancer. N Engl J Med. 2014:371:1963-71.

9. Shaw AT, Solomon BJ. Crizotinib in ROS1-rearranged non-small-cell lung cancer. N Engl J Med. 2015;372:683-4.

10. Takeuchi K, Soda M, Togashi Y, et al. RET, ROS1 and ALK fusions in lung cancer. Nat Med. 2012:18:378-81.

11. Davies KD, Le AT, Theodoro MF, et al. Identifying and targeting ROS1 gene fusions in non-small cell lung cancer. Clin Cancer Res. 2012;18:4570-9.

12. Arai Y, Totoki Y, Takahashi H, et al. Mouse model for ROS1-rearranged lung cancer. PLoS One. 2013;8:e56010.

13. Kato $Y$, Ninomiya $K$, Ohashi $K$, et al. Combined effect of cabozantinib and gefitinib in crizotinib-resistant lung tumors harboring ROS1 fusions. Cancer Sci. 2018:109:3149-58.

14. Kuffer A, Lakkaraju AK, Mogha A, et al. The prion protein is an agonistic ligand of the G protein-coupled receptor Adgrg6. Nature. 2016;536:464-8.

15. Lin JJ, Shaw AT. Recent advances in targeting ROS1 in lung cancer. J Thorac Oncol. 2017:12:1611-25.

16. Lin JJ, Ritterhouse LL, Ali SM, et al. ROS1 fusions rarely overlap with other oncogenic drivers in non-small cell lung cancer. J Thorac Oncol. 2017;12: $872-7$.

17. Wang $W$, Tang $Y, L i ~ J$, et al. Detection of ALK rearrangements in malignant pleural effusion cell blocks from patients with advanced non-small cell lung cancer: a comparison of Ventana immunohistochemistry and fluorescence in situ hybridization. Cancer Cytopathol. 2015;123:117-22.

\section{Publisher's Note}

Springer Nature remains neutral with regard to jurisdictional claims in published maps and institutional affiliations. 